\title{
DIFFUSION OF POTASSIUM ON THE W(112) PLANE PREADSORBED BY Ni ATOMS: FIELD EMISSION CURRENT FLUCTUATIONS AUTOCORRELATION STUDY
}

\author{
T. BIERnat ${ }^{a, *}$, R. BeAszCzyszyN ${ }^{a}$ And Ch. KLeint ${ }^{b}$ \\ ${ }^{a}$ Institute of Experimental Physics, University of Wrocław \\ Pl. Maxa Borna 9, 50-204 Wrocław, Poland \\ ${ }^{b}$ Fakultät für Physik und Geowissenschaften, Universität Leipzig \\ Linnéstr. 5, 7010 Leipzig, Germany \\ (Received December 11, 2000; in final form May 14, 2001)
}

\begin{abstract}
Diffusion of potassium adsorbate on the W(112) plane with preadsorbed nickel was investigated by means of the autocorrelation function of field emission current fluctuations. A comparison of the experimental autocorrelation function with that of a theoretical calculation by Gesley and Swanson gives the surface diffusion coefficient of potassium. From its temperature dependence the diffusion activation energy at several $\mathrm{Ni}$ pre-coverages for a constant potassium coverage $\left(\Theta_{\mathrm{K}}=0.4\right)$ is derived. The dependence of the activation energy for potassium surface diffusion on the nickel coverage - first reduction and then an increase with increasing Ni coverage - is observed. This is in agreement with the results obtained from the spectral analysis of the field emission current fluctuations of the same system. The decrease is understood to be a result of the smoothing effect caused by $\mathrm{Ni}$ atoms on the W(112) plane.
\end{abstract}

PACS numbers: $79.70 .+\mathrm{q}, 68.37 . \mathrm{Vj}, 68.43 . \mathrm{Jk}, 05.40 . \mathrm{Ca}$

\section{Introduction}

Surface diffusion processes can be studied both by monitoring changes in the concentration profile of adsorbates and via examining an adlayer in thermal equilibrium by density fluctuation methods. Reviews on such surface diffusion studies are given in Refs. [1-4].

The density fluctuations of the adsorbate are responsible for the occurrence of field emission (FE) current fluctuations. These fluctuations can be investigated

*corresponding author; e-mail: teresa@ifd.uni.wroc.pl 
by spectral analysis [5] and autocorrelation measurements [6]. Both representations are related by the Wiener-Khintchin theorem, i.e. they are mutual Fourier transforms. The theoretical treatment of the FE current fluctuations carried out by Gesley and Swanson [7] yielded formulas for the spectral density function and the autocorrelation function of the fluctuations. Both representations allow us to calculate the diffusion coefficient by making a comparison of the experimental results with the theoretical curves. Recently the method based on the spectral analysis has been employed in studies of alkali metals [8-12], and the autocorrelation measurements were used to determine the diffusion parameters of hafnium [13] and titanium [14] on the tungsten field emitter surface. The studies have shown that the surface diffusion coefficients and activation energies depend on the plane of a single crystal, crystallographic directions and coverage. As was shown by examining the co-adsorption system W(112) Ni-K [12], even small changes in the structure of the crystal plane influence considerably these parameters.

The present paper contains the results of an autocorrelation study of the FE current fluctuations for the co-adsorption system W(112) Ni-K which was previously examined by the spectral analysis [11, 12]. A comparison of the results obtained using both methods is given. The surface structure changes of the W(112) plane caused by $\mathrm{Ni}$ atomic adsorption on the potassium mobility are discussed.

\section{Experimental}

The investigations of the field emission flicker noise (FEFN) were carried out in a sealed-off field emission microscope tube with a Faraday collector placed behind the probe hole in the screen. The FE current of the probed emitter region of the W(112) plane could be directed to the collector by an external magnetic field. The diameter of the probed region is estimated to be about $10^{-6} \mathrm{~cm}$. The noise signal (FE current fluctuations) after amplification was analysed by a hundred-channel stochastic analyser. The autocorrelation functions were measured. The dc collector currents were controlled to achieve comparable conditions and were kept constant during the course of an experiment. The tube was equipped with an ampoule-type potassium source and a nickel source in the shape of a small $\mathrm{Ni}$ bar spotwelded to two crossed tungsten loops. The residual gas pressure was about $10^{-8} \mathrm{~Pa}$. The emitter temperature was determined by resistance measurements of a loop segment. The experimental arrangement including the electronics was the same as described in Ref. [12]. The measurements of the autocorrelation functions were performed in Leipzig.

\section{Results}

Potassium was deposited on the thermally cleaned W(112) plane with preadsorbed nickel of different amounts, given in deposition minutes $t_{\mathrm{Ni}}$. Figure 1 presents the dependence of the work function $\varphi_{112}$ on the deposition time of nickel $t_{\mathrm{Ni}} \cdot \varphi_{112}$ was calculated from the relation of the voltages for the nickel covered and the clean plane at a constant FE current, $i_{112}=3 \mathrm{nA}$. The curve $\varphi_{112}$ versus nickel coverage has a distinct maximum [15]. The maximum was also observed during the 


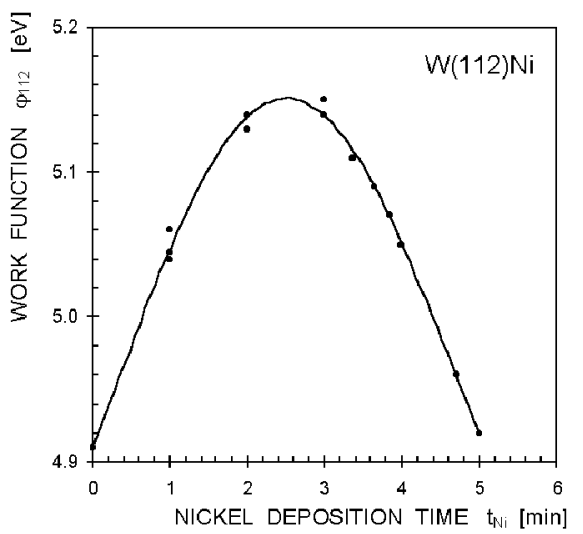

Fig. 1. Work function for the W(112) plane $\varphi_{112}$ versus nickel deposition time $t_{\mathrm{Ni}} \cdot \varphi_{112}$ was calculated from the relation of the voltages for the nickel covered and the clean $W$ plane at a constant $\mathrm{FE}$ current $i_{112}=3 \mathrm{nA}$.

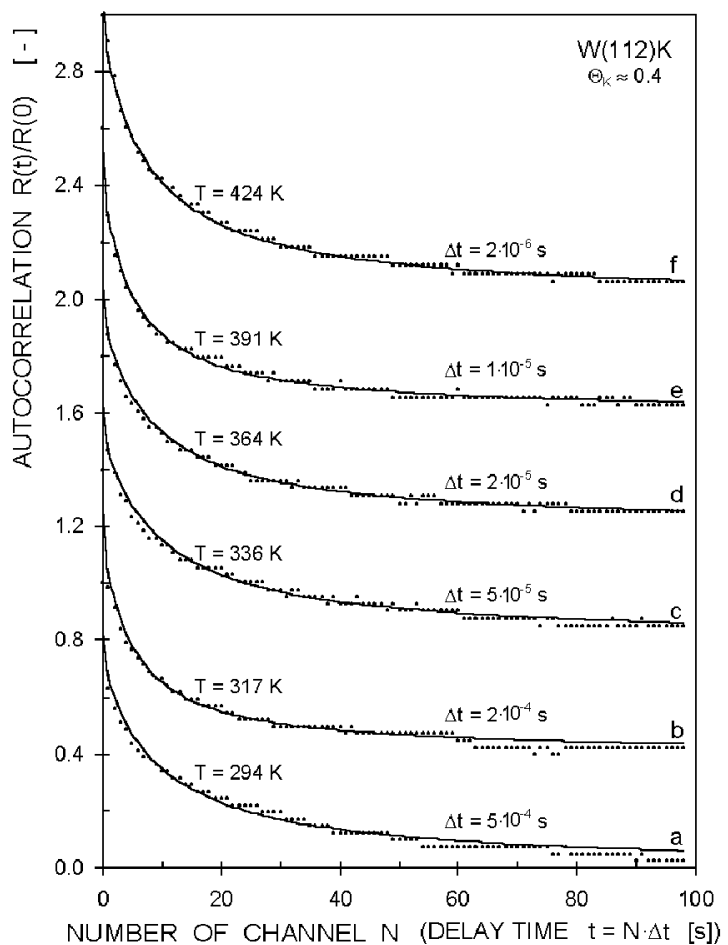

Fig. 2. Autocorrelation functions of the W(112) K system for the average potassium coverage $\Theta \approx 0.4$, on $\mathrm{Ni}$-free tungsten surface, at different substrate temperatures indicated in the figure. Dots correspond to experimental results, solid lines represent theoretical autocorrelation functions of the best fitting to the experimental results. Curves are shifted in the vertical direction by 0.2 each for a better viewing. 


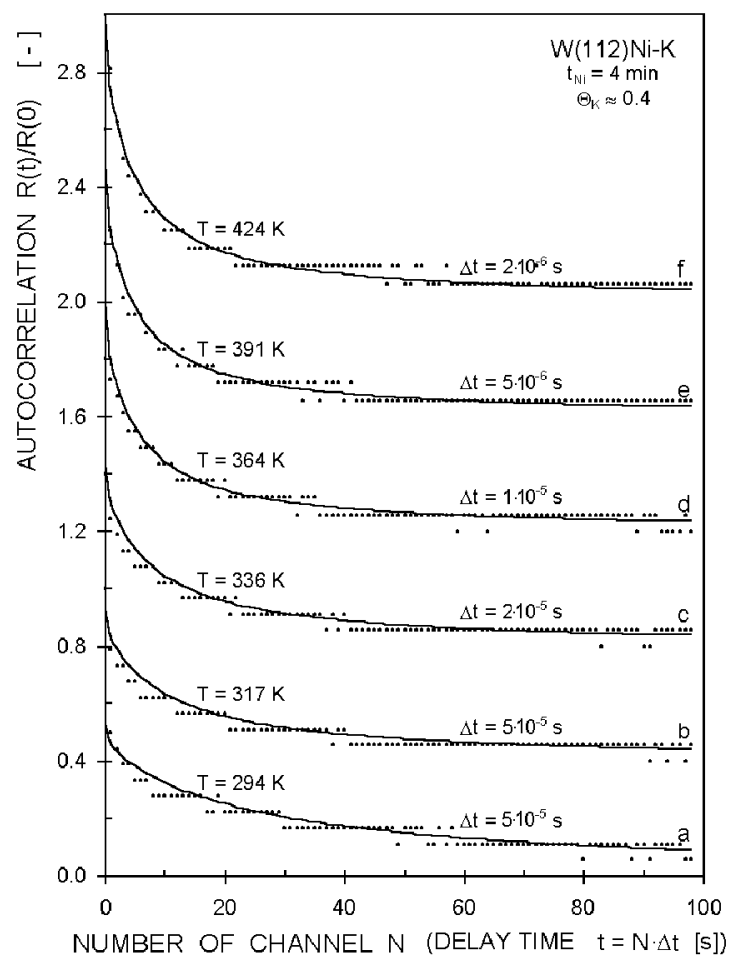

Fig. 3. Autocorrelation functions of co-adsorption layers $\mathrm{Ni}-\mathrm{K}$ on the $\mathrm{W}(112)$ plane. Deposition time of $\mathrm{Ni} t_{\mathrm{Ni}}=4 \mathrm{~min}$, potassium coverage $\Theta_{\mathrm{K}} \approx 0.4$. Emitter temperatures are indicated at the plots. Dots correspond to experimental results, solid lines show theoretical autocorrelation functions of the best fitting to the experimental results. Curves are shifted in the vertical direction by 0.2 each for a better viewing.

investigation by another method [16]. The presence of the maximum is understood to be caused by the smoothing of the W(112) plane by nickel atoms.

The autocorrelation functions were measured at a constant potassium coverage of $\Theta_{\mathrm{K}}=0.4$ in the temperature range of 294-425 K. Figures 2 and 3 show examples of the experimental autocorrelation functions (dots) for a $\mathrm{Ni}$-free surface and for that after a nickel deposition time $t_{\mathrm{Ni}}=4 \mathrm{~min}$, respectively.

\subsection{Determination of the diffusion parameters}

The experimental results are compared with the theoretical autocorrelation function derived by Gesley and Swanson [7] on the assumption that the field emission current fluctuations are induced by fluctuations in the number of adparticles on a small probed area and created by two-dimensional surface diffusion of the adsorbate. The corresponding autocorrelation function for a circular probe region and unbounded diffusion is given by

$$
R(t)=R(0)\left\{1-\exp \left(-2 \alpha^{2}\right)\left[I_{0}\left(2 \alpha^{2}\right)+I_{1}\left(2 \alpha^{2}\right)\right]\right\},
$$


where $\alpha^{2}=t_{0} / t=r_{0}^{2} / 4 D t, r_{0}$ is the radius of the probe region, $D$ is the diffusion coefficient, $I_{0}$ and $I_{1}$ are modified Bessel functions.

Using the least-mean-squares procedure to fit the theoretical curve to the experimental autocorrelation functions the parameter $t_{0}$ corresponding to the best fitting of both curves was determined and the diffusion coefficient was calculated from the relation $t_{0}=r_{0}^{2} / 4 D\left(r_{0}\right.$ is about $5 \times 10^{-7} \mathrm{~cm}$ in this experiment). In Figs. 2 and 3 solid lines represent the theoretical autocorrelation functions of the best fitting to experimental points.

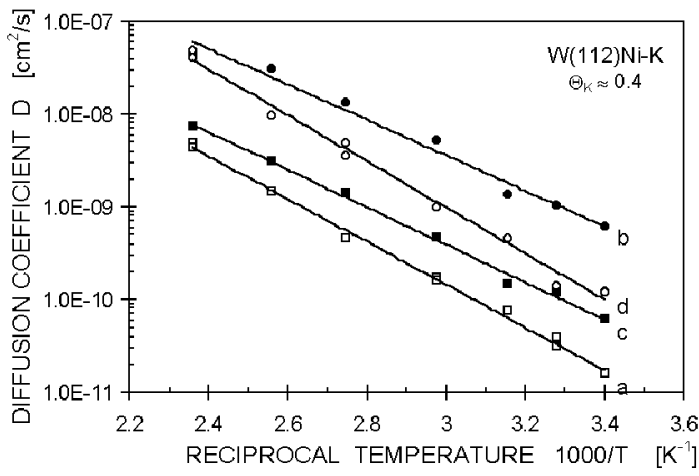

Fig. 4. Temperature dependence of the surface diffusion coefficients for the co-adsorption system $\mathrm{W}(112) \mathrm{Ni}-\mathrm{K}$ at different nickel deposition times. Curves $a, b$, $c, d$ correspond to the deposition times $t_{\mathrm{Ni}}=0,2,4,6 \mathrm{~min}$, respectively. Curves $b$ and $d$ are shifted by one decade for a better viewing.

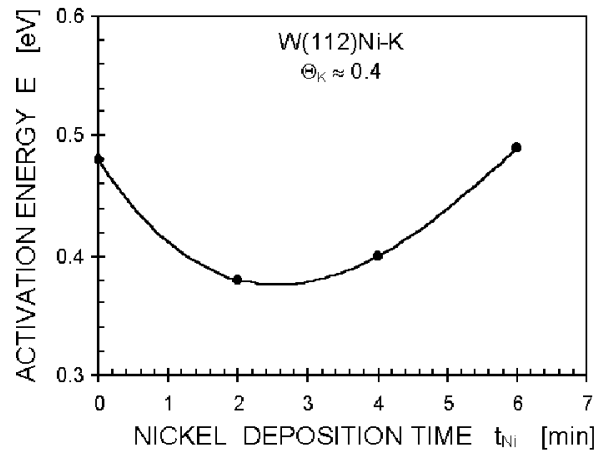

Fig. 5. Activation energy for surface diffusion versus deposition time of nickel determined from the Arrhenius plots in Fig. 4. The solid line is the approximation of the experimental points by a 3rd order polynomial.

Figure 4 provides the Arrhenius plot of the surface diffusion coefficient $\left(D=D_{0} \exp (-E / k T)\right.$ ) for various nickel deposition times. The values of the surface diffusion energy $E$ and pre-exponential factor $D_{0}$ for different nickel coverages are presented in Figs. 5 and 6, respectively. Dots in these figures are experimental points, solid lines are the approximation by 3rd order polynomials. 


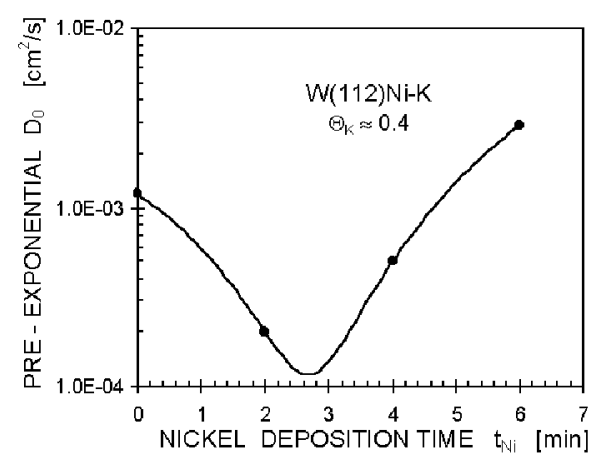

Fig. 6. Nickel coverage dependence of the pre-exponential factor $D_{0}$ determined from the Arrhenius plot in Fig. 4. The solid line is the approximation of the experimental points by a 3rd order polynomial.

\section{Discussion}

The FE current fluctuations of the co-adsorption system W(112) Ni-K were already measured by means of the spectral density functions [11, 12] and cross-correlation functions $[12,17]$ at various temperatures. As was shown in Ref. [17] the current fluctuations of this system are mainly produced by mobility of potassium atoms. Therefore the obtained values of the surface diffusion coefficient $D$ and the activation energy for surface diffusion $E$ can be considered as potassium surface-diffusion parameters.

Figures 2 and 3 demonstrate that the autocorrelation functions depend on the temperature of the substrate and the nickel deposition time. The increase in temperature causes that the autocorrelation functions diminish faster to zero. It can be noticed that the experimental autocorrelation functions are very well fitted by the theoretical functions. This suggests that in the temperature range under investigation we are confronted with one process generated by the adsorbate surface diffusion. Investigations of spectral density functions $[11,12]$ and the cross-correlation functions [17] for the same co-adsorption systems show that at least two different processes: density fluctuations of adsorbate (the Timm and Van der Ziel model [18]) and collective movement of adatoms have to be taken into account for the interpretation of the experimental results. From the autocorrelation measurements this cannot be confirmed. This may be connected with the measurement procedure of the autocorrelation function. The used stochastic analyser allowed only 100 points to obtain for the autocorrelation function.

We assume that surface diffusion understood in terms of density fluctuations plays a dominant role in the adsorbate-induced current fluctuation at the temperature applied. This assumption is supported by the temperature dependence of the diffusion coefficient $D$ derived from fitting the theoretical autocorrelation functions to experimental ones (Fig. 4) which is in agreement with the Arrhenius equation. The activation energy for the surface diffusion $E$ calculated from the slope of the Arrhenius plot depends on the nickel deposition time (Fig. 5). Although the autocorrelation measurement was carried out only for three nickel doses the dependences of the activation energy $E$ and pre-exponential factor $D_{0}$ on 
nickel deposition time show a pronounced minimum at this nickel coverage which corresponds to the maximum of work function $\varphi$ of the W(112) Ni system (see Figs. 1, 5, and 6). On the contrary the dependence of the diffusion coefficient $D$ on $\mathrm{Ni}$ coverage determined for a constant temperature shows a maximum at a $\mathrm{Ni}$ coverage corresponding to the maximum work function $\varphi$ of the system W(112) Ni. It is clear that the surface structure strongly affects the diffusion parameters. As was early found [16], the maximum of the work function is a consequence of the smoothing of the W(112) corrugated plane by nickel atoms. At first the nickel atoms occupy the sites at potential minima along the troughs on the W(112) plane and form one-dimensional islands along the [111] directions, which leads to the increase in the work function. When the nickel atom concentration on the substrate surface is equal to the surface concentration of substrate atoms all trough sites are occupied and the work function $\varphi_{112}$ reaches a maximum. A further rise in $\mathrm{Ni}$ concentration leads to a slight roughening of the topmost layer and consequently to a drop in $\varphi_{112}$. This roughening effect is a result of incorporation of additional $\mathrm{Ni}$ atoms into the Ni rows adsorbed in the $\mathrm{W}$ troughs, which causes the displacement of $\mathrm{Ni}$ atoms from the original adsorption sites. Further Ni deposition leads to adsorption on the ridges of the tungsten rows and subsequently a compression in this layer, which results in a full Ni monolayer of $19.4 \times 10^{14}$ atoms $/ \mathrm{cm}^{2}$ [16].

This smoothing effect is simply reflected in the nickel coverage dependence of $E$ and $D_{0}$. In a simple random-walk model of diffusion, $D_{0}$ is given by the adparticle jump length $l$ and jump attempt frequency $\nu\left(D_{0}=l^{2} \nu\right)$ [1]. While small jumps are possible on a smooth surface, increased roughness should require longer jumps for surface diffusion of the K-adsorbate atoms. This interpretation should be treated as only first approximation of the complex process.

Another point of view [12] should be also taken into consideration in the discussion. During the adsorption of alkali atoms on a metal substrate an electronic charge is transferred from the adatoms to the substrate and a strong interaction between adatoms appears. Such transfer depends on the work function of the substrate [19]. However, the dipole moments of $\mathrm{K}$ atoms as well as lateral and substrate interaction energies will be a complicated function of $\mathrm{Ni}$ coverage. The largest repulsive interaction between the $\mathrm{K}$ adatoms can be expected at the $\mathrm{Ni}$ coverage corresponding to the maximum of the work function of the W(112) Ni system. This should influence the formation of different potassium structures on W(112) face and consequently change the diffusion mechanism and parameters. Such structure-diffusion relations for strongly interacting systems were determined by Kiev group $[1,20]$.

The values of the potassium activation energy for surface diffusion and their nickel deposition-time dependence obtained in this experiment by the autocorrelation function measurements are in agreement with the results obtained by the spectral density function analysis [12] as well as cross-correlation investigations [17]. 


\section{Acknowledgments}

This work was supported by the University of Wroctaw under the Project No. 2016/W/IFD/97. We are indebted to Krzysztof Biernat for the computer programming.

\section{References}

[1] A.G. Naumovets, Yu.S. Vedula, Surf. Sci. Rep. 4, 365 (1984).

[2] R. Gomer, Rep. Prog. Phys. 53, 917 (1990).

[3] Ch. Kleint, Surf. Sci. 200, 472 (1988).

[4] R. Błaszczyszyn, Prog. Surf. Sci. 42, 117 (1993).

[5] Ch. Kleint, Surf. Sci. 25, 394 (1971).

[6] R. Gomer, Surf. Sci. 38, 373 (1973).

[7] M.A. Gesley, L.W. Swanson, Phys. Rev. B 32, 7703 (1985).

[8] T. Biernat, Solid State Phenom. 12, 61 (1990).

[9] J. Bęben, Solid State Phenom. 12, 17 (1990).

[10] T. Biernat, J. Bęben, R. Męclewski, Surf. Sci. 17, 266 (1992).

[11] Ch. Kleint, R. Błaszczyszyn, J. Phys. Colloq. 50, C8-91 (1989).

[12] R. Błaszczyszyn, Ch. Kleint, Surf. Sci. 253, 129 (1991).

[13] J. Bęben, W. Gubernator, Surf. Sci. 304, 59 (1994).

[14] T. Biernat, A.M. Dąbrowski, Vacuum 54, 99 (1999).

[15] M. Błaszczyszyn, R. Błaszczyszyn, A. Ciszewski, Surf. Sci. 304, 325 (1994).

[16] J. Kołaczkiewicz, E. Bauer, Surf. Sci. 144, 495 (1984).

[17] R. Błaszczyszyn, Ch. Kleint, Appl. Phys. A 46, 145 (1988).

[18] G.W. Timm, A. Van der Ziel, Physica 32, 1333 (1966).

[19] K.F. Wojciechowski, Surf. Sci. 80, 253 (1979).

[20] I.F. Lyuksyutov, A.G. Naumovets, W.L. Pokrovsky, Two-Dimensional Crystals, Nautshnaya Dumka, Kiev 1988 (in Russian). 